\title{
Multipole expansion BEM for simultaneous Poisson's equations
}

\author{
T. Matsumoto, T. Takahashi \& S. Taniguchi \\ Department of Mechanical Science and Engineering, \\ Nagoya University, Japan
}

\begin{abstract}
A boundary element method for simultaneous Poisson's equations is presented to solve large scale problems governed by Poisson's equation using multipole expansions of the fundamental solutions. Original Poisson's equation is approximated a set of Poisson's equations and an integral representation for the set of differential equations is derived. The fundamental solutions of the coupled Poisson equations consist of the fundamental solution of Laplace's equation, biharmonic function, and triharmonic function. Multipole expansions of these fundamental solutions are used in the evaluation of the boundary integral equations. The effectiveness of the present formulation is demonstrated through a numerical example.

Keywords: Poisson's equation, fundamental solution, multipole expansion, source distribution.
\end{abstract}

\section{Introduction}

Poisson's equation is a good starting point for analyses of potential problems with inhomogeneous material parameters [1]. The integral representation of Poisson's equation has a domain integral term originated from the source term. To avoid the domain discretization, the domain integral can be converted to boundary integrals by means of the dual reciprocity method (DRM) [2] or the multiple reciprocity method (MRM) [3]. In the DRM, the value of the source term at an arbitrary point in the domain is approximated with a linear combination of radial basis functions (RBF) whose collocation points are placed in the domain and on the boundary. In order to convert the domain integral term originated from the source term of Poisson's equation, particular solutions corresponding to the radial basis function are required. Also, the coefficients of the source term approximation have to be deter- 
mined in advance by collocation method which requires fully populated matrix to solve and is unstable for large scale problems. On the other hand, MRM requires particular solutions for the sources corresponding to a series of fundamental solutions. By using these particular solutions, the original domain integral term can be converted to a series of boundary integrals and a domain integral. Ochiai proposed a variant of MRM, called triple reciprocity BEM [4,5], which applies the reciprocity formulation only three times. In this method, instead of using the correct values of the derivatives of the source, they are roughly estimated to be zero. The error of the derivative of the source on the boundary is modified by using the values of the source at collocation points in the domain instead.

For large-scaled problems, the fast multipole methods (FMM) may also be utilized for those governed by Poisson's equation. To circumvent the evaluation of the domain integrals in applying FMM for Poisson's equation, MRM based approach is more straight-forward in applying FMM, because only the multipole expansions of the higher order fundamental solutions found in the boundary integrals are required in the process.

In this study, we consider Poisson's equation and approximate the source term in terms of simultaneous coupled Poisson's equations. Using the fundamental solutions of the simultaneous Poisson's equations, a set of boundary integral equations, equivalent to those proposed by Ochiai, is derived. The fundamental solutions of the coupled Poisson equations consist of the fundamental solution of Laplace's equation, biharmonic function, and triharmonic function. Multipole expansions of them are used in the evaluation of the boundary integral equations. The resulting set of boundary integral equations are evaluated numerically by using the multipole expansions of the fundamental solutions. The effectiveness of the present formulation is demonstrated through a simple numerical example.

\section{A boundary only integral formulation for Poisson's equation}

Consider a potential problem governed by Poisson's equation

$$
\nabla^{2} \phi_{1}(x)+\phi_{2}(x)=0, \quad x \in V
$$

with the boundary condition

$$
\begin{aligned}
& \phi_{1}(x)=\bar{\phi}_{1}(x), \quad x \in S_{\phi}, \\
& q_{1}(x)=\frac{\partial \phi_{1}(x)}{\partial n}=\bar{q}_{1}(x), \quad x \in S_{q},
\end{aligned}
$$

where $V$ is the domain and $S=S_{\phi} \cup S_{q}$ is its boundary, $\phi_{1}(x)$ denotes the potential and $\phi_{2}(x)$ the source term. Also, $q_{1}(x)=\partial \phi_{1}(x) / \partial n$ is the outward normal derivative of $\phi_{1}(x)$ to the boundary; $\bar{\phi}_{1}$ and $\bar{q}_{1}$ are given functions prescribed on the specified boundaries, respectively.

We assume that the source term $\phi_{2}(x)$ is also assumed to be known both in $V$ and on $S$. 
Now, we assume the source term $\phi_{2}(x)$ is the solution of the following coupled Poisson equations:

$$
\left.\begin{array}{l}
\nabla^{2} \phi_{2}(x)+\phi_{3}(x)=0, \\
\nabla^{2} \phi_{3}(x)+\sum_{k=1}^{M} \alpha^{k} \delta\left(x-z^{k}\right)=0
\end{array}\right\}
$$

where $z^{k}, k=1,2, \ldots, M$ are internal collocation points as shown in Figure 1, and $\alpha^{k}, k=1,2, \ldots, M$ are unknown constants.

The boundary conditions for eqn (4) can be assumed as $[4,5]$

$$
\begin{aligned}
& \phi_{2}(x)=\bar{\phi}_{2}(x), \quad x \in S, \\
& \phi_{3}(x)=-\nabla^{2} \phi_{2}(x)=0, \quad x \in S .
\end{aligned}
$$

By combining eqns (1) and (4), we have a set of Poisson's equations to be solved, as follows:

$$
\left.\begin{array}{l}
\nabla^{2} \phi_{1}(x)+\phi_{2}(x)=0, \\
\nabla^{2} \phi_{2}(x)+\phi_{3}(x)=0, \\
\nabla^{2} \phi_{3}(x)+\sum_{k=1}^{M} \alpha^{k} \delta\left(x-z^{k}\right)=0
\end{array}\right\}
$$

with the conditions as a combination of eqns (2), (3), (5) and (6):

$$
\left.\begin{array}{l}
\phi_{1}(x)=\bar{\phi}_{1}(x), \quad x \in S_{\phi}, \\
q_{1}(x)=\frac{\partial \phi_{1}(x)}{\partial n}=\bar{q}_{1}(x), \quad x \in S_{q}, \\
\phi_{2}(x)=\bar{\phi}_{2}(x), \quad x \in S, V, \\
\phi_{3}(x)=-\nabla^{2} \phi_{2}(x)=0, \quad x \in S .
\end{array}\right\}
$$

The integral representations for the simultaneous Poisson's equations (7) are derived by starting from the following weighted residual form:

$$
\begin{aligned}
& \int_{V} \phi_{1 m}^{*}(x, y)\left(\nabla^{2} \phi_{1}(x)+\phi_{1}(x)\right) d V_{x}+\int_{V} \phi_{2 m}^{*}(x, y)\left(\nabla^{2} \phi_{2}(x)+\phi_{3}(x)\right) d V_{x} \\
& \quad+\int_{V} \phi_{3 m}^{*}(x, y)\left(\nabla^{2} \phi_{3}(x)+\sum_{k=1}^{M} \alpha^{k} \delta\left(x-z^{k}\right)\right) d V_{x}=0
\end{aligned}
$$

where $\phi_{i m}^{*}(x, y)(i, m=1,2,3)$ are weighting functions. 


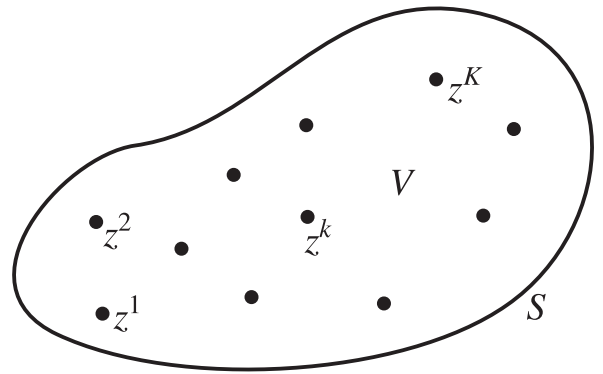

Figure 1: Domain, boundary and internal collocation points.

By integrating eqn (9) by parts repeatedly, we obtain

$$
\begin{aligned}
& \sum_{i=1}^{3}\left\{\int_{S} \phi_{i m}^{*}(x, y) q_{i}(\mathbf{x}) d S_{x}-\int_{S} q_{i m}^{*}(x, y) \phi_{i}(x) d S_{x}\right\} \\
& \quad+\int_{V} \nabla^{2} \phi_{1 m}^{*}(x, y) \phi_{1}(x) d V_{x} \\
& \quad+\int_{V}\left\{\nabla^{2} \phi_{2 m}^{*}(x, y)+\phi_{1 m}^{*}(x, y)\right\} \phi_{2}(x) d V_{x} \\
& \quad+\int_{V}\left\{\nabla^{2} \phi_{3 m}^{*}(x, y)+\phi_{2 m}^{*}(x, y)\right\} \phi_{3}(x) d V_{x}+\sum_{k=1}^{M} \alpha^{k} \phi_{3 m}^{*}\left(z^{k}, y\right)=0 .
\end{aligned}
$$

We now consider particular solutions of the following adjoint form of the original simultaneous Poisson's equations, eqn (4), as the weighting functions $\phi_{i m}^{*}(x, y)$.

$$
\begin{aligned}
& {[L]\left[u^{*}\right]+[I] \delta(\mathbf{x}-\mathbf{y})} \\
& \quad=\left[\begin{array}{ccc}
\nabla^{2} & 0 & 0 \\
1 & \nabla^{2} & 0 \\
0 & 1 & \nabla^{2}
\end{array}\right]\left[\begin{array}{lll}
\phi_{11}^{*} & \phi_{12}^{*} & \phi_{13}^{*} \\
\phi_{21}^{*} & \phi_{22}^{*} & \phi_{23}^{*} \\
\phi_{31}^{*} & \phi_{32}^{*} & \phi_{33}^{*}
\end{array}\right]+[I] \delta(x-y)=0,
\end{aligned}
$$

where $\left[\phi^{*}\right]=\phi_{i m}^{*}(x, y) \mathrm{C}[I]$ denotes the unit matrix and $\delta(x-y)$ is Dirac's delta function.

By applying the property of Dirac's delta function, the second and third terms of eqn (10) becomes

$$
\begin{aligned}
\int_{V} & \nabla^{2} \phi_{1 m}^{*}(x, y) \phi_{1}(x) d V_{x}+\int_{V}\left\{\nabla^{2} \phi_{2 m}^{*}(x, y)+\phi_{1 m}^{*}(x, y)(x)\right\} \phi_{2}(x) d V_{x} \\
& +\int_{V}\left\{\nabla^{2} \phi_{3 m}^{*}(x, y)+\phi_{2 m}^{*}(x, y)(x)\right\} \phi_{3}(x) d V_{x} \\
= & -\phi_{m}(y) .
\end{aligned}
$$


Substituting eqn (12) into (10), we finally obtain an integral representation of eqn (7), as follows:

$$
\begin{aligned}
c(y) \phi_{m}(y)= & \sum_{i=1}^{3}\left\{\int_{S} \phi_{i m}^{*}(x, y) q_{i}(x) d S_{x}-\int_{S} q_{i m}^{*}(x, y) \phi_{i}(x) d S_{x}\right\} \\
& +\sum_{k=1}^{M} \alpha^{k} \phi_{3 m}^{*}\left(z^{k}, y\right)
\end{aligned}
$$

where $c(y)=1$ for $y \in V$ and $c(y)=\frac{1}{2}$ for $y$ located on the smooth part of $S$.

The fundamental solutions $\phi_{i m}^{*}$ are obtained in the form

$$
\left[\phi^{*}\right]=[L]^{c} \Phi(x, y),
$$

where $[L]^{c}$ is the cofactor of $[L]$, and $\Phi(x, y)$ is the fundamental solution for the determinant operator of $[L]$, i.e.,

$$
\operatorname{det}[L] \Phi(x, y)+\delta(x-y)=0 .
$$

Then, finally, $\left[\phi^{*}\right]$ is obtained as follows:

$$
\left[\phi^{*}\right]=\left[\begin{array}{ccc}
\frac{1}{4 \pi r} & 0 & 0 \\
\frac{-r}{8 \pi} & \frac{1}{4 \pi r} & 0 \\
\frac{r^{3}}{96 \pi} & \frac{-r}{8 \pi} & \frac{1}{4 \pi r}
\end{array}\right] .
$$

where $r=|x-y|$.

\section{Discretization of the boundary integral equation}

Discretizing eqn (13) by using $N$ constant elements gives

$$
\begin{aligned}
\frac{1}{2} \phi_{m}\left(y^{l}\right)= & \sum_{i=1}^{3} \sum_{j=1}^{N}\left\{\left(\int_{S_{j}} \phi_{i m}^{*}\left(x, y^{l}\right) d S\right) q_{i}\left(x^{j}\right)\right. \\
& \left.-\left(\int_{S_{j}} q_{i m}^{*}\left(x, y^{l}\right) d S\right) \phi_{i}\left(x^{j}\right)\right\}+\sum_{k=1}^{M} \alpha^{k} \phi_{3 m}^{*}\left(z^{k}, y^{l}\right),
\end{aligned}
$$

where $x^{j}$ and $y^{l}$ denote the centers of geometry of elements $j$ and $l$, respectively.

In eqn (17), the unknowns are either of $\phi_{i}\left(x^{j}\right)$ and $q_{i}\left(x^{j}\right)$, and $\alpha^{k}$, and their total number becomes $3 N+M$.

Although from eqn (17), we obtain $3 N$ equations, we still need $M$ equations in order to solve for $3 N+M$ unknowns. Therefore, we consider additional $M$ 
collocation points in the domain and specify the values for the source term $\phi_{2}\left(z^{k}\right)$, $(k=1, \ldots, M)$. It is achieved by using the component for $m=2$ of eqn (17) and substituting $z^{k},(k=1, \ldots, M)$ for $y^{l}$ as

$$
\begin{aligned}
\phi_{2}\left(z^{l}\right)= & \sum_{i=1}^{3} \sum_{j=1}^{N}\left\{\left(\int_{S_{j}} \phi_{i 2}^{*}\left(x^{j}, z^{l}\right) d S\right) q_{i}\left(x^{j}\right)\right. \\
& \left.-\left(\int_{S_{j}} q_{i 2}^{*}\left(x^{j}, z^{l}\right) d S\right) \phi_{i}\left(x^{j}\right)\right\}+\sum_{k=1}^{M} \alpha^{k} \phi_{32}^{*}\left(z^{k}, z^{l}\right) .
\end{aligned}
$$

The final linear algebraic equations can be written, by collecting all the unknown quantities on the left-hand side, as

$$
[A]\{x\}=\{b\},
$$

where $[A]$ is the coefficient matrix, $\{x\}$ the unknown vector, and $\{b\}$ is the righthand side vector calculated from known quantities. Equation (19) is solved by using the multipole expansions of the fundamental solutions and an iterative solver. The multipole expansion of the components of $\phi_{11}^{*}\left(=\phi_{22}^{*}=\phi_{33}^{*}\right)$ is given as follows:

$$
\begin{aligned}
\frac{1}{4 \pi r} & =\frac{1}{4 \pi|x-y|} \\
& =\frac{1}{4 \pi} \sum_{n=0}^{\infty} \sum_{m=-n}^{n} R_{n, m}(\overrightarrow{O x}) \overrightarrow{S_{n, m}}(\overrightarrow{O y}), \quad|\overrightarrow{O x}|<|\overrightarrow{O y}|,
\end{aligned}
$$

where $R_{n, m}$ and $S_{n, m}$ are defined as [6]

$$
\begin{aligned}
& R_{n, m}(\overrightarrow{O x})=\frac{1}{(n+m) !} P_{n}^{m}(\cos \theta) e^{i m \phi} r^{n}, \\
& S_{n, m}(\overrightarrow{O x})=(n-m) ! P_{n}^{m}(\cos \theta) e^{i m \phi} \frac{1}{r^{n+1}},
\end{aligned}
$$

with the polar coordinates $(r, \theta, \phi)$ of the point $x . P_{n}^{m}$ is the associated Legendre functions. $\phi_{21}^{*}, \phi_{32}^{*}$ and $\phi_{31}^{*}$ are also given as

$$
\begin{aligned}
\phi_{21}^{*}= & \phi_{32}^{*}=-\frac{r}{8 \pi} \\
= & -\frac{1}{8 \pi} \sum_{n=0}^{\infty} \sum_{m=-n}^{n}\left\{\frac{|\overrightarrow{O x}|^{2} R_{n, m}(\overrightarrow{O x}) \overline{S_{n, m}}(\overrightarrow{O y})}{2 n+3} \frac{R_{n, m}(\overrightarrow{O x})|\overrightarrow{O y}|^{2} \overrightarrow{S_{n, m}}(\overrightarrow{O y})}{2 n-1}\right\} \\
\phi_{31}^{*}= & \frac{r^{3}}{96 \pi} \\
= & \frac{1}{96 \pi} \sum_{n=0}^{\infty} \sum_{m=-n}^{n}\left\{\frac{3|\overrightarrow{O x}|^{4} R_{n, m}(\overrightarrow{O x}) \overrightarrow{S_{n, m}}(\overrightarrow{O y})}{(2 n+3)(2 n+5)}\right. \\
& \left.-\frac{6|\overrightarrow{O x}|^{2} R_{n, m}(\overrightarrow{O x})|\overrightarrow{O y}|^{2} \overline{S_{n, m}}(\overrightarrow{O y})}{(2 n-1)(2 n+3)}+\frac{3 R_{n, m}(\overrightarrow{O x})|\overrightarrow{O y}|^{4} \overrightarrow{S_{n, m}}(\overrightarrow{O y})}{(2 n-3)(2 n-1)}\right\} .
\end{aligned}
$$




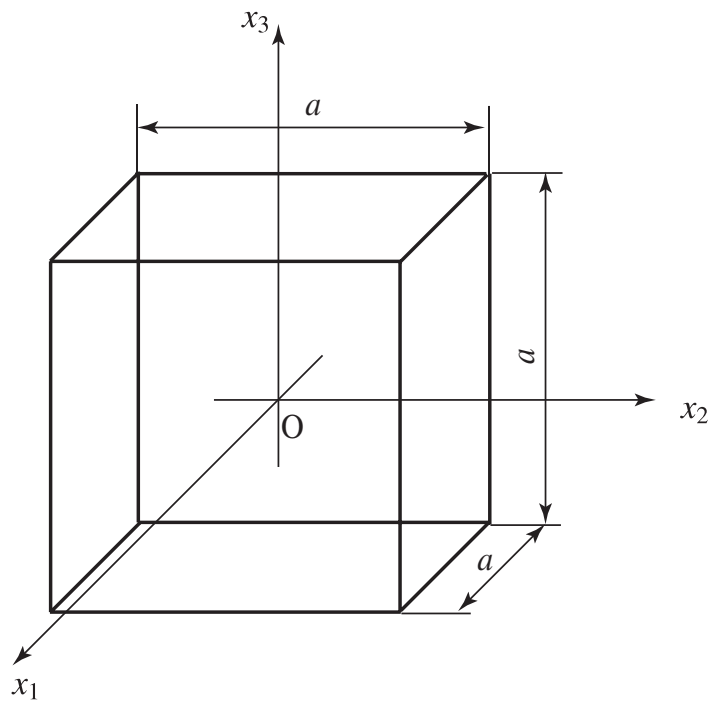

Figure 2: A cube model.

\section{Numerical example}

Consider a cubic region having an edge of $a=10$ as shown in Figure 2. All sides of the cube are divided uniformly into 3072 triangular constant elements, and 45 internal collocation points are placed as shown in Figure 3. The boundary condition for $\phi_{1}(x)$ and the known distribution of $\phi_{2}(x)$ are given by

$$
\begin{aligned}
& \phi_{1}(x)=\left\{\begin{array}{l}
0 \quad \text { at } x_{3}=-5 \\
100 \text { at } x_{3}=5
\end{array},\right. \\
& q_{1}(x)=0 \text { at } x_{1}, x_{2}= \pm 5, \\
& \phi_{2}(x)=10 \sin \left(\frac{\pi}{5} x_{3}+\pi\right) .
\end{aligned}
$$

The infinite series of the multipole expansions of the fundamental solutions are truncated at 7 th terms and GMRES is used as the iterative solver with the convergence criterion $\varepsilon=10^{-5}$.

Figure 4 shows the results for $\phi_{1}\left(x_{3}\right)$ at the internal collocation points. The results obtained by the present approach show good agreements with the exact solutions.

\section{Concluding remarks}

A boundary element method for Poisson's equation has been presented. The source term is approximated with simultaneous coupled Poisson's equations. The funda- 
10 Mesh Reduction Methods

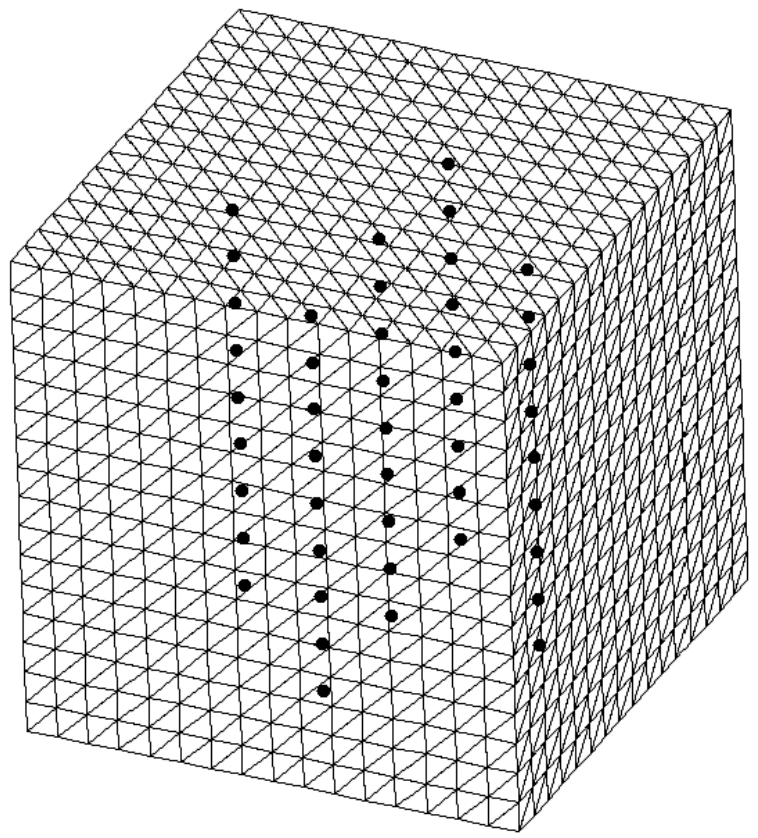

Figure 3: Boundary elements and internal collocation points.

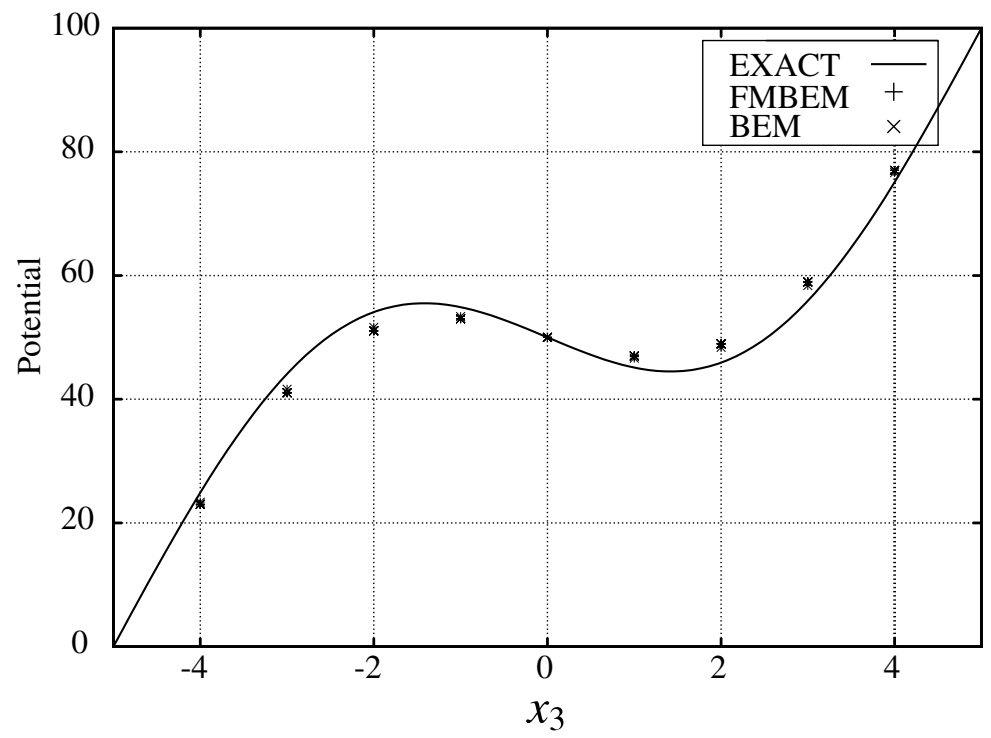

Figure 4: Result for $\phi_{1}$ at internal points. 
mental solutions of the coupled Poisson's equations have been derived and the corresponding boundary integral equation has been presented. The fundamental solutions of the coupled Poisson equations consist of the fundamental solution of Laplace's equation, biharmonic function, and triharmonic function. Multipole expansions of these fundamental solutions have been used in the evaluation of the boundary integral equations. The numerical test example has demonstrated the effectiveness of the present approach.

\section{References}

[1] Matsumoto, T., Guzik, A. \& Tanaka, M., A boundary element method for analysis of thermoelastic deformations in materials with temperature dependent properties International Journal for Numerical Methods in Engineering, 64(11), pp. 1432-1458, 2005.

[2] Partridge, P.W., Brebbia C.A. \& Wrobel, L.C., The Dual Reciprocity Boundary Element Method, Computational Mechanics Publications, Southampton, 1992.

[3] Nowak, A.J., The Multiple Reciprocity Boundary Element Method, Neves, A.C. (Ed.), Computational Mechanics Publications, Southampton, 1994.

[4] Ochiai, Y., Three-dimensional steady thermal stress analysis by triplereciprocity boundary element method, International Journal for Numerical Methods in Engineering, 63(12), pp. 1741-1756, 2005.

[5] Ochiai, Y., Sladek, V. \& Sladek, J., Transient heat conduction analysis by triple-reciprocity boundary element method, Engineering Analysis with Boundary Elements, 30(3), pp. 194-204, 2006.

[6] Yoshida, K., Applications of Fast Multipole Method to Boundary Integral Equation Method, PhD Thesis, Kyoto Univ., Japan, 2001. 